\title{
Survival benefit of neoadjuvant chemotherapy in pathologic T2NO or lower urothelial carcinoma patients: evidence to support the use of neoadjuvant chemotherapy
}

\author{
Hyeong Dong Yuk, Chang Wook Jeong, Cheol Kwak, Hyeon Hoe Kim, Ja Hyeon Ku \\ Department of Urology, Seoul National University Hospital, Seoul, Korea \\ Contributions: (I) Conception and design: HD Yuk, JH Ku; (II) Administrative support: HH Kim, C Kwak, CW Jeong; (III) Provision of study \\ materials or patients: HH Kim, C Kwak, CW Jeong; (IV) Collection and assembly of data: HD Yuk; (V) Data analysis and interpretation: HD Yuk; (VI) \\ Manuscript writing: All authors; (VII) Final approval of manuscript: All authors. \\ Correspondence to: Ja Hyeon Ku, MD, PhD. Department of Urology, Seoul National University Hospital, 101 Daehak-ro, Jongno-gu, Seoul 110-744, \\ Korea. Email: kuuro70@snu.ac.kr.
}

\begin{abstract}
Background: To evaluate the survival benefit of neoadjuvant chemotherapy (NAC) in pathologic T2N0 or less patients.

Methods: A total of 526 patients with less than pT2N0 underwent radical cystectomy. Patients were divided into three groups: non-NAC, those who did not receive NAC; partial NAC, those who received less 3 cycles of NAC; and complete NAC, those who received 3 cycles of NAC.

Results: Median follow up was 74.6 (range, 24-311) months. Recurrent-free survival (RFS) was significantly $(\mathrm{P}=0.041)$ higher in the non-NAC group than that in the complete NAC group. Overall survival (OS) was significantly $(\mathrm{P}=0.039)$ higher in the non-NAC group than that in the complete NAC group. There was no significant difference between the partial NAC group and the complete NAC group. In patients with pT0, the NAC group had higher pT0 ratio than the non-NAC group (33.3\% vs. $21.1 \%$ ). A total of $66.6 \%$ of NAC patients were down-staged to less than T2. In univariate and multivariate analyses, recurrence was significantly related to pathologic $\mathrm{T}$ stage $(\mathrm{P}<0.001)$, concurrent carcinoma in situ $(\mathrm{CIS})(\mathrm{P}=0.002)$, and the number of removed lymph nodes $(\mathrm{LNs})(\mathrm{P}=0.001)$. Survival was significantly related to pathologic $\mathrm{T}$ stage $(\mathrm{P}<0.001)$, lymphovascular invasion $(\mathrm{LVI})(\mathrm{P}=0.002)$, the number of removed $\mathrm{LNs}(\mathrm{P}<0.001)$, and the presence of NAC $(\mathrm{P}=0.047)$.
\end{abstract}

Conclusions: Patients with pT2 or lower underwent NAC showed similar prognosis as patients with pT2 or lower who did not undergo NAC.

Keywords: Bladder cancer; chemotherapy; neoadjuvant chemotherapy (NAC); radical cystectomy; urothelial carcinoma (UC)

Submitted Nov 05, 2019. Accepted for publication Feb 14, 2020.

doi: 10.21037/tau-19-705

View this article at: http://dx.doi.org/10.21037/tau-19-705

\section{Introduction}

Bladder cancer is one of the most common cancers of the urogenital system (1). About $25 \%$ of patients diagnosed with bladder cancer for the first time are diagnosed with muscle invasive bladder cancer (MIBC). Of patients diagnosed with non-muscle invasive bladder cancer (NMIBC), about 20$30 \%$ progress to $\mathrm{MIBC}(2,3)$. MIBC is an aggressive disease with high risk of metastasis and poor prognosis (4). The standard treatment for MIBC is radical cystectomy with pelvic lymph node dissection (PLND) $(5,6)$. Despite radical cystectomy, 10-year cancer-specific survival (CSS) rate after radical cystectomy for MIBC patients is only about $67 \%$ (7). In the case of node positive patients, the 10 -year CSS rate is about $17 \%$ regardless of the stage of the primary tumor (7). 
Many patients tend to relapse after surgery probably due to micro-metastasis at or before radical cystectomy $(8,9)$. To improve these results, neoadjuvant chemotherapy (NAC) has been used since the 1980s (10). It has been shown that cisplatin-based NAC can increase overall survival (OS) by $5-8 \%$ (11). NAC is recommended for patients with clinical T2, T3, or T4a N0M0 in several guidelines $(11,12)$. In the Southwest Oncology Group (SWOG) Intergroup study, radical cystectomy after NAC improved complete pathologic response by $23 \%$ and median OS by 31 months compared with cystectomy alone (5). In a randomized control study for cT2-4N0 patients, NAC and radical cystectomy improved the OS by $16 \%$ and complete response by $21.2 \%$ compared to radical cystectomy alone (6). However, the role of NAC in patients under T2 is controversial. The purpose of this study was to compare the clinical course differences in patients with pathologic T2N0M0 or lower after NAC and those with pathologic T2N0M0 or less who did not undergo NAC.

\section{Methods}

The Institutional Review Board of Seoul National University Hospital approved this retrospective study and waived the requirement to obtain informed consent from patients. All research and related protocols used in this study complied with the principles of the Declaration of Helsinki. We retrospectively reviewed medical records of patients who underwent radical cystectomy with bladder cancer from January 1991 to December 2016. Patients with T2 or less N0M0 were included in this study. All patients underwent radical cystectomy and standard PLND or extended PLND. Only pathologically confirmed patients with urothelial carcinoma (UC) were included. Patients with previous chemotherapy history except NAC were excluded, Patients with previous other cancer treatment or with metastasis were also excluded. Patients were divided into two groups: those who received NAC and those who did not. TNM stage and tumor grade were classified according to the 2010 American Joint Committee on Cancer classification and the 2004 World Health Organization/ International Society of Urologic Pathology consensus classifications. We collected information such as patient age, body mass index (BMI), sex, American Society of Anesthesiologists (ASA) physical status, clinical TNM stage, pathological TNM stage, margin positive, number of lymph node (LN) removed, whether to perform NAC, number of NAC cycles, and various oncologic results including recurrence and mortality. In the absence of recurrence or metastasis, most patients underwent a routine test every 3 months for 3 years after surgery and every 6 months for 4 to 5 years. Thereafter, routine test was conducted once a year. Routine test included laboratory test, urine test, urine cytology, and bladder cystoscopy. Patients with neo-bladder included a post-voided residual urine check using ultrasonic bladder scan. Radiological test such as chest computed tomography (CT), abdomen-pelvis CT, and bone scan were performed once a year. NAC used cisplatin and gemcitabine regimen or methotrexate, vinblastine, doxorubicin, and cisplatin (MVAC) regimen. The number of NAC cycles was 2 or 3. Continuous variables are expressed as means and standard deviations (SDs) or median values and intermediate ranges (IQRs) while categorical variables are expressed by the frequency of events (\%). Primary endpoint was OS. Secondary endpoint was recurrence-free survival (RFS). All survival results were analyzed by Kaplan survival analysis and log-rank test. Cox regression analysis was used to analyze factors associated with various oncologic outcomes. All statistical tests were performed using IBM SPSS Statistics, version 22.0 (IBM, Armonk, NY, USA) and STATA version 14 (StataCorp LP, College Station, TX, USA). A $\mathrm{P}$ value of less than 0.05 was considered to indicate statistical significance.

\section{Results}

\section{Baseline patient characteristics}

A total of 857 patients underwent radical cystectomy with bladder cancer from January 1991 to December 2016 in a tertiary institution. All patients with pathologic findings of UC and less than pT2N0M0 were included in this study. The total number of patients included in the study was 526. Patients were divided into three groups according to the presence or absence of NAC: (I) non-NAC, those who did not receive NAC; (II) partial NAC, those who received less 3 cycles of NAC; and (III) complete NAC, those who received 3 cycles of NAC.

The partial NAC group had 40 patients and the complete NAC group had 35 patients. The non-NAC group had 451 patients. Table 1 shows characteristics of these patients. Clinical T stage was cT2 in most patients of the nonNAC group. It was cT3 in more than $60 \%$ of patients in the partial NAC group and $65.7 \%$ in the complete NAC group $(\mathrm{P}=0.003)$. It could be seen that NAC was applied to the group with higher tumor burden. Complete 
Table 1 Patient characteristics according to NAC

\begin{tabular}{|c|c|c|c|c|}
\hline Variables & Non-NAC (N=451) & Partial NAC (N=40) & Complete NAC $(\mathrm{N}=35)$ & $P$ value \\
\hline $\mathrm{BMI}\left(\mathrm{kg} / \mathrm{m}^{2}\right)$ & $22.9 \pm 3.9$ & $23.3 \pm 3.2$ & $24.0 \pm 3.2$ & 0.086 \\
\hline Sex & & & & 0.851 \\
\hline Men & $383(84.9)$ & $35(87.5)$ & $29(82.9)$ & \\
\hline ASA classification & & & & 0.156 \\
\hline 1 & $159(35.3)$ & $15(37.5)$ & $14(40.0)$ & \\
\hline 2 & $259(57.4)$ & $19(47.5)$ & $21(60.0)$ & \\
\hline$\geq 3$ & $33(7.3)$ & $6(15.0)$ & 0 & \\
\hline $\mathrm{T} 2$ & $275(60.9)$ & $15(37.5)$ & $10(28.6)$ & \\
\hline T3 & $8(1.8)$ & $25(62.5)$ & 25 (71.4) & \\
\hline Clinical N stage & & & & 0.652 \\
\hline No & $451(92.6)$ & $40(97.6)$ & $35(89.7)$ & \\
\hline $\mathrm{N} 1$ & $19(3.9)$ & 0 & $1(2.6)$ & \\
\hline $\mathrm{N} 2$ & $17(3.5)$ & $1(2.4)$ & $3(7.7)$ & \\
\hline Pathologic T stage & & & & 0.393 \\
\hline TO & $95(21.1)$ & $15(37.5)$ & $10(28.6)$ & \\
\hline Low & $32(7.1)$ & $1(2.5)$ & 0 & \\
\hline High & $315(69.8)$ & $27(67.5)$ & $23(65.7)$ & \\
\hline Margin positive & $6(1.3)$ & 0 & 0 & 0.187 \\
\hline LVI & $66(14.6)$ & $2(5.0)$ & $4(11.4)$ & 0.218 \\
\hline Concurrent CIS & $66(14.6)$ & $2(5.0)$ & 4 (11.4) & 0.218 \\
\hline Removed LN number & $13.9 \pm 12.3$ & $13.8 \pm 10.4$ & $12.3 \pm 13.3$ & 0.479 \\
\hline Recurrence & $109(24.2)$ & $9(22.5)$ & $13(37.1)$ & 0.217 \\
\hline Overall mortality & $146(32.7)$ & $7(17.5)$ & $13(37.1)$ & 0.112 \\
\hline
\end{tabular}

Data was present as $n(\%)$ or mean \pm SD. NAC, neoadjuvant chemotherapy; BMI, body mass index; ASA, American Society of Anesthesiologists; LVI, lymphovascular invasion; CIS, carcinoma in situ; LN, lymph node; SD, standard deviation. 
A

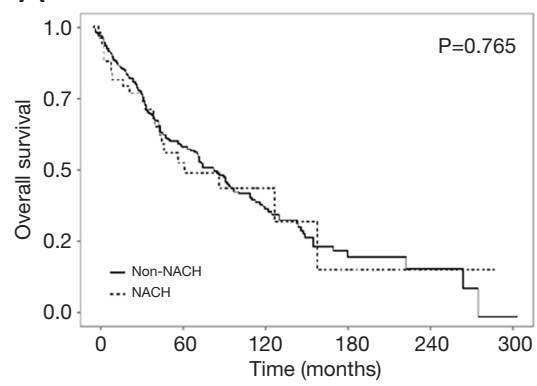

B

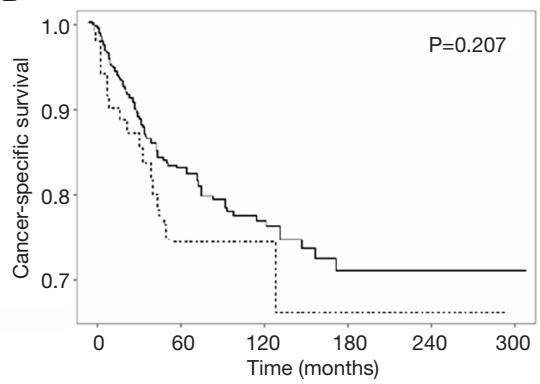

C

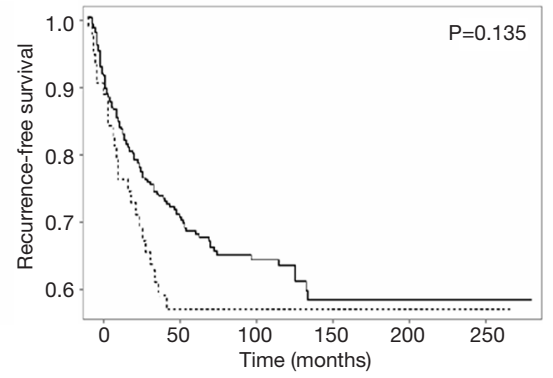

Figure 1 Oncologic outcomes according to NAC. (A) OS; (B) CSS; (C) RFS. NAC, neoadjuvant chemotherapy; OS, overall survival; CSS, cancer-specific survival; RFS, recurrence-free survival.

A

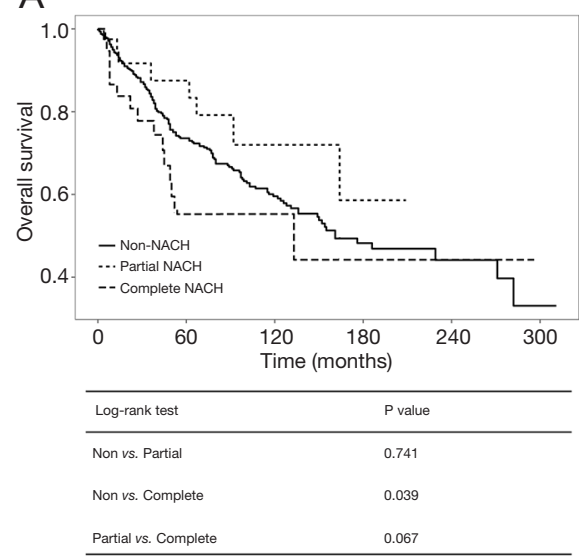

B

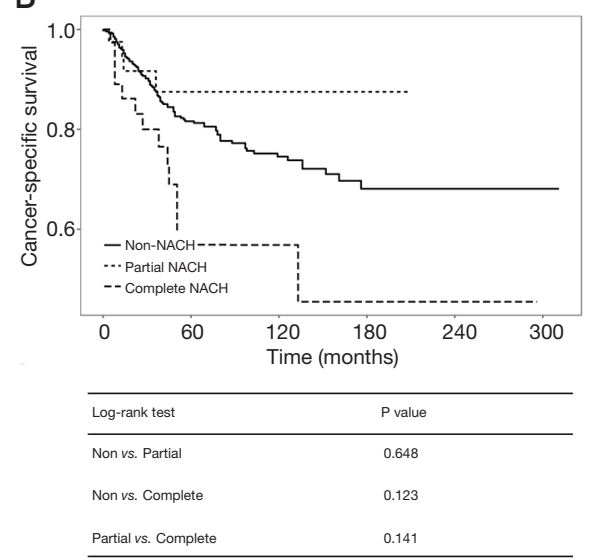

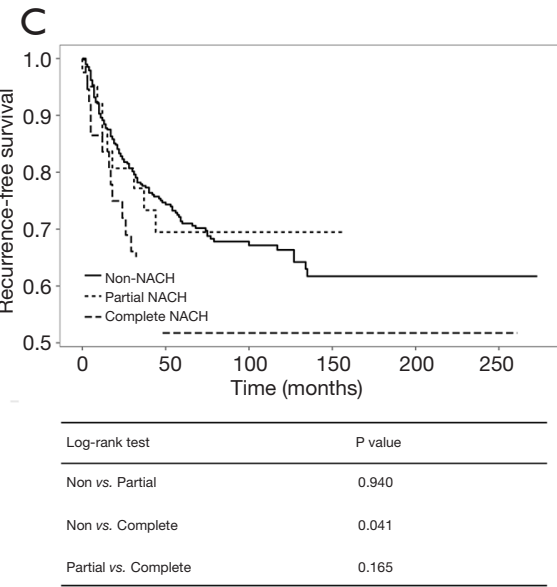

Figure 2 Oncologic outcomes according to NAC cycle. (A) OS; (B) CSS; (C) RFS. NAC, neoadjuvant chemotherapy; OS, overall survival; CSS, cancer-specific survival; RFS, recurrence-free survival.

pathologic response was achieved in $21.1 \%$ of the nonNAC group, $37.5 \%$ of the partial NAC group, and $28.6 \%$ of the complete NAC group. Complete pathologic response rate was higher in the NAC group than that in the nonNAC group, although the difference was not statistically significant (Table 1).

\section{Survival outcomes according to NAC}

There was no difference in the survival results between the NAC group and non-NAC group (Figure 1). Comparison of survival outcome between partial NAC and complete NAC and non-NAC group, the non-NAC group had better $(\mathrm{P}=0.039)$ prognosis than the complete NAC group in OS. In CSS, there was no significant difference in prognosis between complete NAC and non-NAC groups. There was no significant difference in OS or CSS between partial NAC and non-NAC groups or between partial NAC and complete NAC groups. In RFS, the non-NAC group had better $(\mathrm{P}=0.041)$ prognosis than the complete NAC group. There was no significant difference in prognosis between partial NAC and non-NAC groups or between partial NAC and complete NAC groups (Figure 2).

\section{Multivariate Cox proportional hazards analyses of NAC on survival outcomes}

In multivariate Cox proportional hazards analysis, age, pathologic $\mathrm{T}$ stage, lymphovascular invasion (LVI), number of removed LNs, and NAC showed significant associations with OS. Age, pathologic T stage, LVI, concurrent carcinoma in situ (CIS), number of removed LNs, and NAC were significant factors associated with 
Table 2 Multivariate Cox proportional hazards analyses of NAC on OS, CSS and RFS

\begin{tabular}{|c|c|c|c|c|}
\hline Variables & \multicolumn{2}{|l|}{ OS } & \multicolumn{2}{|c|}{ RFS } \\
\hline Age & $1.047(1.025-1.070)$ & $<0.001$ & $0.999(0.981-1.018)$ & 0.943 \\
\hline \multicolumn{5}{|l|}{ Sex } \\
\hline Women & Reference & & Reference & \\
\hline NAC & & 0.047 & & 0.158 \\
\hline None & Reference & & Reference & \\
\hline Partial & $0.451(0.206-0.985)$ & 0.046 & $0.592(0.343-1.024)$ & 0.061 \\
\hline Complete & $0.217(0.059-0.795)$ & 0.021 & $0.723(0.322-1.623)$ & 0.411 \\
\hline Tis & $1.236(0.418-3.654)$ & 0.701 & $1.403(0.567-3.469)$ & 0.464 \\
\hline $\mathrm{Ta}$ & $1.881(0.897-3.947)$ & 0.180 & $1.423(0.893-3.929)$ & 0.032 \\
\hline $\mathrm{T} 1$ & $2.522(1.233-5.158)$ & 0.095 & $1.664(0.879-3.150)$ & 0.118 \\
\hline $\mathrm{T} 2$ & $1.236(0.418-3.654)$ & 0.011 & $2.136(1.151-3.961)$ & 0.016 \\
\hline Tumor grade & & 0.423 & & 0.717 \\
\hline Low & Reference & & Reference & \\
\hline High & $0.880(0.373-2.075)$ & 0.770 & $0.715(0.312-1.638)$ & 0.428 \\
\hline LVI & 1.195 (1.168-1.913) & 0.001 & $1.612(0.921-2.822)$ & 0.094 \\
\hline
\end{tabular}

NAC, neoadjuvant chemotherapy; OS, overall survival; CSS, cancer-specific survival; RFS, recurrence-free survival; HR, hazard ratio; CI, confidence interval; LVI, lymphovascular invasion; CIS, carcinoma in situ; LN, lymph node.

CSS. Pathologic T stage, concurrent CIS, and number of removed LNs were significant factors associated with RFS (Table 2).

\section{Univariate and multivariate logistic regression models of predictive factors of pathologic T2NO or lower after NAC}

The difference between NAC cycle and regimen was not related to pT2N0 or lower after NAC. In univariate and multivariate logistic regression analysis, clinical $\mathrm{T}$ stage, LVI showed significant associations with pathologic LVI was poor predict factor of pT2N0 or lower after NAC. However, concurrent CIS was a good predict factor (Table 3).

\section{Discussion}

The purpose of NAC before radical cystectomy is to reduce preoperative microinvasion and recurrence (distant recurrence and locoregional recurrence) (13). In several guidelines, NAC is recommended for patients with T2-T4a NOM0 $(2,12)$ because microinvasion is thought to be higher in T3 or T4 with more tumor burden than T2 with smaller tumor burden (14). In the present study, the percentage of high stage above cT3 was higher in the partial and complete NAC groups than that in the non-NAC group. NAC has several advantages. One of them is that NAC affects pathologic status, enabling $\mathrm{pT} 0$ or $\mathrm{pN} 0$ postoperatively and enabling negative surgical margin $(5,13)$. In clinical $T$ stage, 
Table 3 Predictive factors of pathologic T2N0 or lower after NAC according to univariate and multivariate logistic regression models

\begin{tabular}{|c|c|c|c|c|}
\hline Variables & \multicolumn{2}{|c|}{ Univariate } & \multicolumn{2}{|c|}{ Multivariate } \\
\hline Age & $1.017(0.995-1.055)$ & 0.293 & $0.997(0.987-1.001)$ & 0.395 \\
\hline \multicolumn{5}{|l|}{ Sex } \\
\hline Women & Reference & & Reference & \\
\hline NAC regimens & & 0.085 & & 0.203 \\
\hline GC & Reference & & Reference & \\
\hline MVAC & $2.048(1.083-3.872)$ & 0.027 & $1.858(0.820-4.213)$ & 0.138 \\
\hline Others & $1.037(0.622-1.728)$ & 0.890 & $1.522(0.791-2.929)$ & 0.209 \\
\hline \multicolumn{5}{|l|}{ NAC } \\
\hline Complete & $0.880(0.191-4.050)$ & 0.870 & $1.002(0.285-3.525)$ & 0.997 \\
\hline Clinical T stage & & 0.032 & & 0.037 \\
\hline $\mathrm{T} 2$ & Reference & & Reference & \\
\hline T3 & $0.984(0.667-1.025)$ & 0.325 & $0.320(0.164-1.079)$ & 0.170 \\
\hline $\mathrm{T} 4$ & $0.237(0.182-0.675)$ & 0.023 & $0.189(0.075-0.852)$ & 0.021 \\
\hline Tumor grade & & 0.423 & & 0.717 \\
\hline Low & Reference & & Reference & \\
\hline High & $0.422(0.116-1.538)$ & 0.191 & $0.863(0.281-2.649)$ & 0.797 \\
\hline
\end{tabular}

NAC, neoadjuvant chemotherapy; HR, hazard ratio; Cl, confidence interval; LVI, lymphovascular invasion; CIS, carcinoma in situ.

cT2 and cT3 in NAC-administered group was higher than in the non-NAC group. However, in pathologic T stage after surgery, the ratio of T0 in NAC-administered group was similar to or higher than that in the non-NAC group (Table 1).

In $\mathrm{OS}$, the non-NAC group had better $(\mathrm{P}=0.039)$ prognosis than the complete NAC group. The complete NAC group had similar or slightly worse prognosis than the non-NAC group. In RFS, the complete NAC group had worse $(\mathrm{P}=0.041)$ prognosis than the non-NAC group. In this study, NAC had no significant gain in RFS. However, in OS, there was a gain through NAC. In clinical T stage, the NAC group had higher tumor burden than the non-NAC group. NAC was performed for patients with relatively high tumor burden. Although NAC groups had relatively higher tumor burden, their OS was similar to or slightly worse than the non-NAC group. Proportions of pathologic T0 and negative resection margin were also higher in NAC groups. Results showed that the complete NAC group had worse OS than the non-NAC group. Thus, NAC seemed to be ineffective. However, in the preoperative clinical stage, complete NAC group had higher burden in pathologic status, yet there was no significant difference in OS. This indicated that NAC was effective even for groups with stage below T2 (Figure 1).

In a study of MD Anderson's clinical risk stratification, patients with pT0-4N0/+ were included. And patients were divided into high- and low-risk groups according to hydroureteronephrosis, LVI, micropapillary or neuroendocrine features, and cT3b-4a stage. The 5-year survival rate for low-risk patients with RC without NAC was $68 \%(15)$. In other studies, the 5 -year survival rate of 
low-risk patients who underwent RC without NAC ranged from $77 \%$ to $100 \%(9,16)$. Based on these studies, the authors recommend $\mathrm{ACH}$ after RC rather than NAC in low-risk groups $(9,15,16)$.

However, in Mayo Clinic's study of 1,931 patients, patients with pT0-4N0/+ were included. And patients were divided into high and low-risk groups according to hydronephrosis, LVI, histologic variant, and cT3-4 stage. NAC patients had better prognosis than non-NAC patients. Five-year CSS rate was $68 \%$ for NAC patients and $50 \%$ for non-NAC patients $(\mathrm{P}=0.001)$ (17). The OS of non-NAC patients was $67.3 \%$, which was lower than that $(73.3 \%)$ of NAC patients. The proportion of pT0 [OR: 3.05, 95\% confidence interval (CI): 1.89-4.93, $\mathrm{P}<0.001]$ after $\mathrm{RC}$ was significantly higher in the NAC group. The proportion of pT2 or lower (OR: 2.53, 95\% CI: 1.64-3.89, P<0.001) after RC was also significantly higher in the NAC group (17). In our study, the CSS rate was $75.4 \%$ in the NAC group and $72.4 \%$ in the non-NAC group. The NAC group had higher pT0 ratio than the non-NAC group (33.3\% vs. $21.1 \%)$. At baseline, $66.6 \%$ of NAC patients had cT3 or higher stage. However, there was no pT3 or higher patient after NAC and RC. Thus, $66.6 \%$ of NAC patients were down-staged to less than T2 after NAC and RC.

Our research has several limitations. First, it was a retrospective study rather than a randomized study. Second, the significant difference may be insufficient for T0 downstaging with various oncological outcomes and survival rate because it is a prime number of NAC group compared to non-NAC group. Third, those who had missing data were included in this study. Due to the nature of this retrospective study, some patients did not receive NAC for preoperative low renal function or had poor performance status. This might lead to some bias for the result of survival outcome. Fourth, we considered NAC without considering previous TURB or intravesical treatment. Fifth, there may be diversity or limitations of potential interoperability with data from a single center.

\section{Conclusions}

NAC is eligible for low-risk patients with stage below pT2N0. NAC showed favorable results in T0 down-staging and below pT2 downstaging. A patient with a high tumor burden prior to NAC treatment showed similar prognosis to patients with a low tumor burden after NAC. This will serve as a basis for appropriate treatment selection for MIBC patients.

\section{Acknowledgments}

Funding: This work was supported by a Basic Science Research Program through the National Research Foundation of Korea (NRF) funded by the Ministry of Education (NRF-2019R1F1A1050507).

\section{Footnote}

Conflicts of Interest: All authors have completed the ICMJE uniform disclosure form (available at http://dx. doi. org/10. 21037/tau-19-705). JHK serves as an unpaid editorial board member of Translational Andrology and Urology from Jan 2020 to Dec 2021. The other authors have no other conflicts of interest to declare.

Ethical Statement: The authors are accountable for all aspects of the work in ensuring that questions related to the accuracy or integrity of any part of the work are appropriately investigated and resolved. The study was approved by the Institutional Review Board of Seoul National University Hospital (H-1811-108-987) and waived the requirement to obtain informed consent from patients.

Open Access Statement: This is an Open Access article distributed in accordance with the Creative Commons Attribution-NonCommercial-NoDerivs 4.0 International License (CC BY-NC-ND 4.0), which permits the noncommercial replication and distribution of the article with the strict proviso that no changes or edits are made and the original work is properly cited (including links to both the formal publication through the relevant DOI and the license). See: https://creativecommons.org/licenses/by-nc-nd/4.0/.

\section{References}

1. Bray F, Ferlay J, Soerjomataram I, et al. Global cancer statistics 2018: GLOBOCAN estimates of incidence and mortality worldwide for 36 cancers in 185 countries. CA Cancer J Clin 2018;68:394-424.

2. Babjuk M, Böhle A, Burger M, et al. EAU guidelines on non-muscle-invasive urothelial carcinoma of the bladder: update 2016. Eur Urol 2017;71:447-61.

3. Guillaume L, Guy L. Epidemiology of and risk factors for bladder cancer and for urothelial tumors. Rev Prat 2014;64:1372-4, 1378-80.

4. Alfred Witjes J, Lebret T, Compérat EM, et al. Updated 2016 EAU Guidelines on Muscle-invasive and Metastatic 
Bladder Cancer. Eur Urol 2017;71:462-75.

5. Grossman HB, Natale RB, Tangen CM, et al. Neoadjuvant chemotherapy plus cystectomy compared with cystectomy alone for locally advanced bladder cancer. N Engl J Med 2003;349:859-66.

6. International Collaboration of Trialists, Medical Research Council Advanced Bladder Cancer Working Party (now the National Cancer Research Institute Bladder Cancer Clinical Studies Group), European Organisation for Research and Treatment of Cancer Genito-Urinary Tract Cancer Group, et al. International phase III trial assessing neoadjuvant cisplatin, methotrexate, and vinblastine chemotherapy for muscle-invasive bladder cancer: longterm results of the BA06 30894 trial. J Clin Oncol 2011;29:2171-7.

7. Stein JP, Skinner DG. Radical cystectomy for invasive bladder cancer: long-term results of a standard procedure. World J Urol 2006;24:296-304.

8. Hautmann RE, Gschwend JE, de Petriconi RC, et al. Cystectomy for transitional cell carcinoma of the bladder: results of a surgery only series in the neobladder era. $\mathrm{J}$ Urol 2006;176:486-92; discussion 491-2.

9. Stein JP, Lieskovsky G, Cote R, et al. Radical cystectomy in the treatment of invasive bladder cancer: long-term results in 1,054 patients. J Clin Oncol 2001;19:666-75.

10. David KA, Milowsky MI, Ritchey J, et al. Low incidence of perioperative chemotherapy for stage III bladder cancer 1998 to 2003: a report from the National Cancer Data

Cite this article as: Yuk HD, Jeong CW, Kwak C, Kim HH, $\mathrm{Ku} \mathrm{JH}$. Survival benefit of neoadjuvant chemotherapy in pathologic T2N0 or lower urothelial carcinoma patients: evidence to support the use of neoadjuvant chemotherapy. Transl Androl Urol 2020;9(3):1270-1277. doi:10.21037/tau-19705
Base. J Urol 2007;178:451-4.

11. Witjes JA, Compérat E, Cowan NC, et al. EAU guidelines on muscle-invasive and metastatic bladder cancer: summary of the 2013 guidelines. Eur Urol 2014;65:778-92.

12. Flaig TW, Spiess PE, Agarwal N, et al. NCCN guidelines insights: bladder cancer, version 5.2018. J Natl Compr Canc Netw 2018;16:1041-53.

13. Zargar H, Espiritu PN, Fairey AS, et al. Multicenter assessment of neoadjuvant chemotherapy for muscleinvasive bladder cancer. Eur Urol 2015;67:241-9.

14. Sherif A, Holmberg L, Rintala E, et al. Neoadjuvant cisplatinum based combination chemotherapy in patients with invasive bladder cancer: a combined analysis of two Nordic studies. Eur Urol 2004;45:297-303.

15. Culp SH, Dickstein RJ, Grossman HB, et al. Refining patient selection for neoadjuvant chemotherapy before radical cystectomy. J Urol 2014;191:40-7.

16. Moschini M, Soria F, Klatte T, et al. Validation of preoperative risk grouping of the selection of patients most likely to benefit from neoadjuvant chemotherapy before radical cystectomy. Clin Genitourin Cancer 2017;15:e267-73.

17. Lyon TD, Frank I, Sharma V, et al. A risk-stratified approach to neoadjuvant chemotherapy in muscle-invasive bladder cancer: implications for patients classified with low-risk disease. World J Urol 2019;37:1605-13. 